\title{
Transmitter Optimization and Theoretical Bounds for Dispersion-Limited Optical Fiber Links
}

\author{
Roberto Gaudino, Member, IEEE, and Emanuele Viterbo, Member, IEEE
}

\begin{abstract}
In this paper, we present a novel mathematical investigation on the dispersion-limited optical communication channel. We tackle the problem using a comprehensive approach that is based on the optimization of the energy transfer from the input to the output of the channel. We solve the optimization problem deriving a fundamental integral equation, which we are able to solve analytically. We show that the dispersion-limited optical channel has a very interesting equivalence to the ideal lowpass filter. This equivalence allows us to derive new bounds on the maximum achievable bit rate on the channel with limited intersymbol interference (ISI). In particular, we demonstrate that by suitably increasing the memory of the modulator and using the optimal pulses derived in this paper, one can transmit with limited ISI over a channel with arbitrarily high dispersion.
\end{abstract}

Index Terms-Optical fiber bandwidth, optical fiber communication, optical fiber dispersion, optical modulation, optical pulse shaping, pulse-shaping methods.

\section{INTRODUCTION}

$\mathbf{M}$ ANY RECENT papers in optical communications focus on finding optical modulation formats that enable extending the maximum achievable distance when transmitting over standard single-mode fibers (SMF), where chromatic dispersion is usually the most important physical impairment. In metropolitan area networks (MANs), most fibers are still SMF and, due to cost limitation, the use of optical dispersion compensation is to be avoided as much as possible. This motivates today's great interest in fully understanding the characteristic of the so-called "dispersion-limited" optical channel, i.e., a channel where chromatic dispersion significantly distorts the transmitted signal, generating high levels of intersymbol interference (ISI). In fact, the problem to be solved for this channel is the minimization of ISI at the receiver.

In this MAN and Extended-MAN scenario, the current trend is to try to increase the dispersion limit by operating on the electronics of either the transmitter (through new modulation formats, [4], [8]) or the receiver (through electrical equalization [11]), without increasing the optical complexity of the system, and in particular, without using special optical filtering or dispersion compensation. Several papers have been published on duobinary/phase-shaped binary transmission (PSBT) optical modulation [8], a line-coding technique that allows more than doubling the dispersion limit with respect to

Paper approved by R. Hui, the Editor for Optical Transmission and Switching of the IEEE Communications Society. Manuscript received October 7, 2003; revised February 9, 2004.

The authors are with the PhotonLab, Dipartimento di Elettronica, Politecnico di Torino, 10129 Torino, Italy (e-mail: roberto.gaudino@polito.it; emanuele.viterbo@polito.it).

Digital Object Identifier 10.1109/TCOMM.2004.833187 standard not-return-to-zero (NRZ) optical modulation, without any modification on the receiver side. Duobinary is now implemented in several precommercial optical transceivers. Other authors, such as [4], proposed more advanced line coding, or other techniques, such as chirped-return-to-zero (CRZ) [1] or carrier-suppressed RZ (CSRZ) [7].

In this paper, we propose a novel analysis of the dispersionlimited optical channel with two main targets. On one side, we find the expression for the optimal transmitted signal maximizing the energy transfer over the dispersive channel. On the other side, using the same criteria, we derive bounds on the maximum achievable bit rate over the dispersion-limited optical channel, when a given maximum amount of ISI is tolerated. Moreover, our results on the optimal pulses may lead in the future to a better understanding of optimal optical line coding and/or to practical implementations.

Some preliminary results on this topic appeared in our previous paper [6]. Here we present the full mathematical formulation of the problem, its analytical solution, and new interesting results on the dispersion-limited optical-channel theoretical bounds. Moreover, in [6], we only considered memoryless transmitters, while now we extend the results to modulation schemes with memory (line coding).

The paper is organized as follows. In Section II, we formulate the optimization problem, and we present its closed-form solution, showing its equivalence to a well-known canonical problem related to the minimization of the time-bandwidth product of a signal [12]. In Section III, we derive, starting from the closed-form solution, an interesting upper bound to the maximum achievable bit rate over the dispersion-limited optical channel, relating it to the memory of the transmitted modulation scheme. In Section IV, we show the potential applications of our theory in realistic optical links. We conclude in Section V with a final discussion of our results.

\section{A. Assumptions}

The results in this paper are based on some basic assumptions consistent with the typical constraints imposed by today's optical technology. In particular, we will analyze the following scenario.

- The optical transmitter is based on an ideal continuous wave $(\mathrm{CW})$ laser and on external modulation. We assume to modulate independently the amplitude and phase of each individual pulse corresponding to a given bit. This is a reasonable assumption, since several of the previously cited modulation formats (CRZ and CSRZ) are today implemented using the cascade of (at least) two independently driven amplitude and phase modulators. 


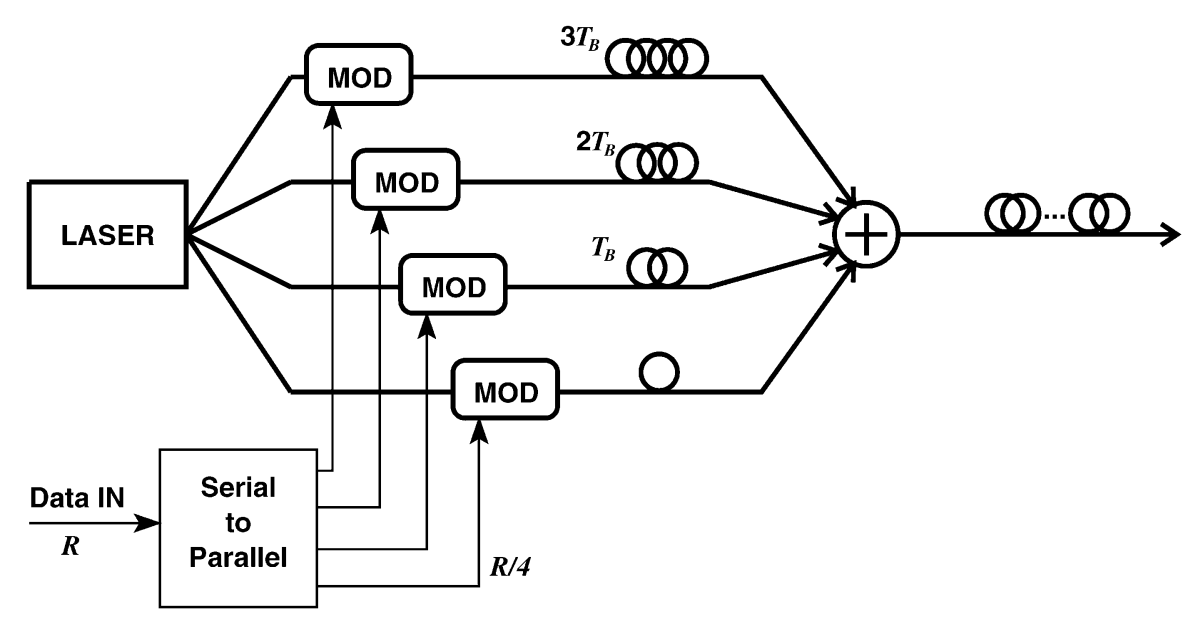

Fig. 1. A possible practical implementation of the modulator with memory, for $n_{\text {mem }}=4$.

- The transmission is binary on-off keying (OOK), and we do not consider multilevel modulation. We will consider transmission with memory, i.e., setups where the pulse transmitted for an individual bit can be arbitrary longer than the bit duration. Thus, we basically assume implementing optical line coding or, equivalently, partial-response signaling. Optical line coding is today commonly implemented in a very simple form in optical duobinary transmission [8]. The idea of modulation with memory is a key issue in this paper, and will be explained in detail in Section I-B.

- Chromatic dispersion is the only impairment in the optical link and no optical dispersion compensation is present. The channel we consider is thus characterized by a single parameter, namely, its accumulated dispersion.

- The receiver is a standard direct-detection optical receiver: a large optical filter, followed by a photodiode and an electrical filter, without electronic compensation [11]. We assume single-threshold detection, and symbol-by-symbol detection, i.e., decisions are taken on each individual bit without taking memory into account. This means that the information, at the receiver side, is only carried by the power of the received signal, while its phase information is lost.

\section{B. Optical Modulation With Memory}

All the above assumptions match well to today's high performance, externally modulated optical links, with the exception of the idea of modulation with memory. Though well known in other communication areas under such names as partial-response signaling or line coding, memory modulation is not yet widespread in optical communication. It is not a totally new concept, since optical duobinary modulation, which is recently gaining significant attention, can be interpreted as a form of line coding, as shown, for example, in [9].

Using modulation with memory, the pulse transmitted for each individual bit (or symbol, if the transmission is multilevel) has a duration that extends beyond the one-bit window. $T_{B}$ being the bit duration, the pulse transmitted for each individual bit will have a duration $T_{\text {in }}=n_{\mathrm{mem}} \cdot T_{B}$, where the (integer) parameter $n_{\text {mem }}$ is usually called the "transmitter" memory. As a practical example, a system working at $10 \mathrm{Gbit} / \mathrm{s}\left[T_{B}=100\right.$ picoseconds (ps)] with $n_{\text {mem }}=4$ will use pulses at the transmitter side with a duration $T_{\text {in }}=400 \mathrm{ps}$. The particular case $n_{\text {mem }}=1$ corresponds to a standard, memoryless modulation. For $n_{\text {mem }}>1$, it should be noted that the signal coming out of the transmitter is affected by ISI. Anyway, line coding is usually associated with a propagation channel that, under suitable conditions, reduces or cancels the amount of ISI present at the transmitter. For instance, optical duobinary can be interpreted as line coding with $n_{\text {mem }}=2$ [9]. In fact, the resulting duobinary signal at the transmitter output is strongly affected by ISI, giving rise to a three-level eye diagram. In the duobinary case, the ISI at the transmitter is cancelled by the direct-detection receiver, which converts the three-level ISI-affected signal into a standard two-level signal without ISI. In general, in line coding, a controlled amount of ISI is created at the transmitter in order to have some kind of advantage at the receiver.

The practical implementation of a generic optical modulator with memory is outside the scope of this paper, which is mainly focused on finding the theoretical transmission bounds of the dispersion-limited channels. A schematic of a possible realization of a modulator with $n_{\mathrm{mem}}=4$ is proposed in Fig. 1. It is an extension of the optical time-domain multiplexing principle [10]. Let us assume again that we are dealing with a system at $R=10 \mathrm{Gbit} / \mathrm{s}\left(T_{B}=100 \mathrm{ps}\right)$ with $n_{\mathrm{mem}}=4$. The light coming from a CW laser is split into $n_{\mathrm{mem}}=4$ fibers. Each fiber feeds an optical modulator that receives the data from a 1:4 serial-to-parallel converter, so that the input data stream for each modulator is actually at a rate $R / 4(2.5 \mathrm{Gbit} / \mathrm{s})$. The key point is that, using this approach, the pulses that each modulator should generate do not overlap in time, since the available time window for each pulse is $4 T_{B}=400 \mathrm{ps}$, so that the modulation on each of the four branches becomes a standard memoryless modulation at $2.5 \mathrm{Gbit} / \mathrm{s}$. Each modulator is then followed by an optical delay line with delay equal to $0, T_{B}, 2 T_{B}$, and $3 T_{B}$, and by an optical coupler that recombines the signals.

\section{Mathematical Assessment of the Problem}

Being interested in dispersion-limited systems, we focus on a fiber transmission model which includes first-order chromatic dispersion only, neglecting all other transmission impairments. 
Thus, we consider the well-known fiber-dispersive transfer function [4]

$$
H_{F}(f)=e^{-j \frac{\beta_{2}}{2} L(2 \pi f)^{2}}
$$

where $\beta_{2}=-(D) /\left(2 \pi f_{0}\right)$ is the chromatic-dispersion parameter, $D$ being the fiber-chromatic dispersion (usually expressed in ps $/ \mathrm{nm} / \mathrm{km}$ ) and $f_{0}$ the central frequency, while $L$ is the fiber length. As commonly accepted, we will indicate as асситиlated dispersion the quantity $\beta_{2} L$ in $\mathrm{ps}^{2}$, or equivalently, $D L$ in $\mathrm{ps} / \mathrm{nm}$.

In order to simplify the expressions, we introduce the normalized dispersion index (NDI) $\gamma,{ }^{1}$ defined as

$$
\gamma=-2 \beta_{2} L R^{2}
$$

where $R=1 / T_{B}$ is the system bit rate $\left(T_{B}\right.$ being the bit duration). The $\gamma$ parameter is quite useful in simplifying the equations, normalizing them to the system bit rate $R$ or the bit duration $T_{B}$. In fact, using this notation, the transfer function becomes

$$
H_{F}(f)=e^{j \gamma\left(\frac{\pi f}{R}\right)^{2}}
$$

while the impulse response of the fiber is given by [4]

$$
h_{F}(t)=\frac{e^{j \pi / 4 \operatorname{sign}(\gamma)}}{T_{B} \sqrt{\pi|\gamma|}} e^{-\frac{j}{\gamma}\left(\frac{t}{T_{B}}\right)^{2}} .
$$

We assume that the transmitted binary digital signal (at the optical level) is in the form

$$
x(t)=\sum_{k=-\infty}^{+\infty} \alpha_{k} \cdot s_{\text {in }}\left(t-k T_{B}\right)
$$

where $s_{\text {in }}(t)$ is the complex envelope of the transmitted pulse for a single bit, and $\alpha_{k}$ assumes the values 0 and 1 for a standard OOK modulation. Since the channel is linear and time invariant (LTI), the resulting pulse at the fiber output is $s_{\text {out }}(t)=s_{\text {in }}(t) *$ $h_{F}(t)$. The goal of our paper is the optimization of the input pulse $s_{\text {in }}(t)$ under the following assumptions.

- The input pulse $s_{\text {in }}(t)$ is strictly time limited to the interval $I=\left[-T_{\text {in }} / 2,+T_{\text {in }} / 2\right]$. As a particular case, we have $T_{\text {in }}=T_{B}$ for a standard memoryless transmitter, but we will show that the case $T_{\text {in }}>T_{B}$, corresponding to a transmitter with memory, is extremely interesting in extending the dispersion limit. In particular, as already mentioned in Section I-B, we will assume $T_{\text {in }}=n_{\text {mem }}$. $T_{B}$, where the (integer) parameter $n_{\text {mem }}$ is the transmitter memory.

- We chose as optimization criterion the maximization of the energy transfer from an input time window $I$ to an output time window $J=\left[-T_{\text {out }} / 2,+T_{\text {out }} / 2\right]$. More specifically, we introduce the input and output energies

$$
\begin{aligned}
\mathcal{E}_{\text {in }} & =\int_{I}\left|s_{\text {in }}(t)\right|^{2} d t \\
\mathcal{E}_{\text {out }} & =\int_{J}\left|s_{\text {out }}(t)\right|^{2} d t
\end{aligned}
$$

${ }^{1}$ The $\gamma$ parameter has already been used by other authors, such as [4]. This $\gamma$ should not be confused with the optical-fiber nonlinear Kerr coefficient. and we maximize over $s_{\text {in }}(t)$ the energy transfer ratio (ETR), defined as

$$
\mathrm{ETR}=\frac{\mathcal{E}_{\text {out }}}{\mathcal{E}_{\text {in }}} .
$$

The pulses $s_{\text {in }}(t)$ resulting from the optimization process proposed here will be indicated as "optimal pulses" in the rest of the paper. Since the impulse response in (4) is a even function of $t$, then it can be shown that the input-output optimal pulses are also even functions of $t$ [3], justifying our assumption of a symmetric output time window $J$.

- The criterion we have chosen is particularly relevant for the case $T_{\text {out }}=T_{B}$, since we assume symbol-by-symbol detection for a binary memoryless receiver (i.e., a receiver taking decisions on single received bits). The concentration of the output pulse energy over a $T_{B}$ time window is effective in both minimizing ISI (which is the goal of our paper) and in increasing the signal-to-noise ratio (SNR) at the decision instant for any "reasonable" digital receiver. In fact, the criterion is "exact" for an ideal optical integrate-and-dump receiver, since in this case, the decision sample is directly proportional to the signal energy over a $T_{B}$ time window. Anyway, as we will show a posteriori in Section IV, it proves an extremely good criterion for realistic optical receiver structures. We notice that for $n_{\text {mem }}>1$, we are considering a somehow nonintuitive system where ISI is strongly present at the transmitter side, but then it is reduced, or even cancelled, by the propagation over the dispersive channel.

- We will show, again in Section IV, that the ETR (for $T_{\text {out }}=T_{B}$ ) for realistic optical receivers should typically be above $90 \%$ to give a penalty due to ISI in the $1-2 \mathrm{~dB}$ range. As a consequence, we will conventionally define in the rest of the paper the "dispersion limit" as the amount of accumulated dispersion for a given bit rate that results in an $\mathrm{ETR}=90 \%$.

\section{A. Fundamental Parameters and Equations}

The ETR optimization problem over a generic LTI system is a canonical problem that was studied in the past [3], [5], and can be reduced to the optimization of a quadratic functional in $s_{\text {in }}(t)$, with a quadratic constraint, as recalled in the Appendix. For a generic filter-impulse response, it leads to the following Fredholm integral equation of the second kind:

$$
\int_{I} \mathcal{K}(u, v) s_{\text {in }}(u) d u=\lambda s_{\text {in }}(v)
$$

where the kernel of the integral equation is

$$
\mathcal{K}(u, v)=\int_{J} h_{F}(z-u) h_{F}^{*}(z-v) d z
$$

and where the optimal solution is given by the eigenfunction corresponding to the maximum eigenvalue $\lambda$, which is equal to the ETR (8), as shown in the Appendix.

This problem has been solved in the literature for several types of bandlimited and standard lowpass filters [3], [5], [12]. In this paper, we solve it (for the first time to our knowledge) 
considering the fiber-dispersive transfer function (1) as the bandlimiting filter. In this case, replacing (4) in (10), by straightforward calculations, the kernel can be expressed as

$$
\mathcal{K}(u, v)=\frac{e^{-j\left(\frac{u^{2}-v^{2}}{\gamma T_{B}^{2}}\right)}}{\pi(u-v)} \cdot \sin \left[\frac{T_{\text {out }}(u-v)}{|\gamma| T_{B}^{2}}\right] .
$$

\section{B. Numerical Solutions}

The resulting integral equation is well conditioned, and can be solved by a suitable numerical method. We used the method described in [3, Sec. IV.A], which simply discretizes time over an $n \times n$ grid $\left[\tau_{i}, \tau_{j}\right]$, and reduces the integral equation (9) to a simple (and numerically stable) discrete eigenvalue problem of the form

$$
\sum_{i=1}^{n} \mathcal{K}\left(\tau_{i}, \tau_{j}\right) s_{\mathrm{in}}\left(\tau_{i}\right) \Delta \tau_{i}=\lambda s_{\mathrm{in}}\left(\tau_{j}\right), \quad j=1, \ldots, n .
$$

Most of the numerical results shown in Section IV were obtained using this numerical solution.

\section{Closed-Form Solution}

The integral equation (9), with the kernel (11), can be solved by looking for a solution in the form $s_{\text {in }}(t)=a(t) \cdot e^{j \phi(t)}$, where $a(t)$ and $\phi(t)$ are real functions of time. This separates the two input-pulse contributions that are usually called amplitude modulation and phase modulation (or chirp). In particular, we look for a solution of the form

$$
s_{\mathrm{in}}(t)=a(t) \cdot e^{\frac{j}{\gamma}\left(\frac{t}{T_{B}}\right)^{2}} .
$$

This "guess" was originally driven by the observation of the numerical results obtained in [6], and proved to be exact, as shown in the following.

By writing the kernel as $\mathcal{K}(u, v)=\mathcal{K}_{\mathcal{R}}(u, v) \cdot \exp$ $\left[-j\left(\left(u^{2}-v^{2}\right) /\left(\gamma T_{B}^{2}\right)\right)\right]$, where

$$
\mathcal{K}_{\mathcal{R}}(u, v)=\frac{1}{\pi(u-v)} \cdot \sin \left[\frac{T_{\text {out }}(u-v)}{|\gamma| T_{B}^{2}}\right]
$$

and by substituting (13) into (9), the phase terms vanish, and the resulting integral equation simplifies to

$$
\int_{I} \mathcal{K}_{\mathcal{R}}(u, v) a(u) d u=\lambda a(v) .
$$

The same integral equation results from the ETR pulse optimization over an ideal lowpass filter with bandwidth $W$ and $J=[-\infty,+\infty]$. This problem received a lot of attention in the past, in the framework of fundamental works on communications theory, and it was fully analyzed and analytically solved in [12]. It leads to an integral equation with kernel

$$
\mathcal{K}_{L P}(u, v)=\frac{\sin [2 \pi W(u-v)]}{\pi(u-v)} .
$$

Thus, the integral equation (15) giving the amplitude $a(t)$ is mathematically equivalent to the ideal lowpass case. A full treatment of these results can be found in [13], [14], or in [15], where the expression of the result in terms of prolate spheroidal functions is given.
We conclude that the solutions of the form (13) are unique, and the one corresponding to the the maximum eigenvalue of (15) corresponds to the one maximizing the ETR.

By direct comparison between the kernels (14) and (16), we observe that

$$
2 \pi W=\frac{T_{\text {out }}}{|\gamma| T_{B}^{2}} \Rightarrow W=\frac{T_{\text {out }}}{2 \pi|\gamma| T_{B}^{2}} .
$$

This is, in our opinion, the most important result of this paper, not only because it gives a closed-form formulation, (i.e., the solution for the ideal lowpass case expressed through prolate spheroidal functions [12]), but even more because it leads to the interesting results we illustrate in the following section.

\section{OPtimal ChIRP AND ChanNel-EQuivalent BANDWIDTH}

The previous results lead to the following important considerations.

- The optimal pulses have a phase modulation given by $\phi(t)=\left(t^{2}\right) /\left(\gamma T_{B}^{2}\right)$, or equivalently, $\phi(t)=$ $-\left(t^{2}\right) /\left(2 \beta_{2} L\right)$. This expression gives the optimal chirp for pulses launched over a dispersive channel. Interestingly, this result was already found in [4], using a totally different approach for which optimality was not proven.

- Provided that the pulse chirp is chosen to be optimal, the dispersive channel is totally equivalent, at least in the ETR sense, to an ideal lowpass filter with bandwidth

$$
W=\frac{T_{\text {out }}}{2 \pi|\gamma| T_{B}^{2}}, \text { or equivalently, } W=\frac{1}{4 \pi} \frac{T_{\text {out }}}{\left|\beta_{2}\right| L} .
$$

This result can be usefully interpreted as a definition of the equivalent bandwidth of the dispersive channel which, to our knowledge, was never given before in a rigorous form. We note here that dispersive channel-transfer function (1) has a peculiar expression that renders most of the common bandwidth definitions totally useless, since $\left|H_{F}(f)\right|^{2}=$ $1 \forall f$. For instance, the commonly used noise-equivalent bandwidth is infinite, and the 3-dB bandwidth is meaningless.

- In the ideal lowpass problem, it can be shown that the ETR depends only on $W T_{\mathrm{in}}$, and the function ETR $=f\left(W T_{\mathrm{in}}\right)$ is monotonically increasing, asymptotically reaching ETR $=1$ for $W T_{\text {in }} \rightarrow+\infty$ [12]. If we fix the limiting value ETR $=0.9$ (a 90\% energy transfer, see discussion in Section IV), the condition $W T_{\text {in }} \geq 0.675$ must be satisfied [12]. In our case, using (18), the ETR is a function of $\left(T_{\text {out }} T_{\text {in }}\right) /\left(2 \pi|\gamma| T_{B}^{2}\right)$ only. If we fix $T_{\text {out }}=T_{B}$ and $T_{\mathrm{in}}=n_{\mathrm{mem}} \cdot T_{B}$, we have that the ETR is only a function of $n_{\text {mem }} /|\gamma|$.

- In order to have ETR $=0.9$, for $n_{\text {mem }}=1$, we have the condition $|\gamma| \leq 0.236$, or equivalently, introducing (2)

$$
R^{2} \leq \frac{0.1179}{\left|\beta_{2} L\right|}
$$

This last equation can be interpreted as the theoretical upper bound to the maximum bit rate that can be achieved over the dispersive optical channel with limited ISI (i.e., 
TABLE I

MaXimum ACCEPTABLE ACCUMUlated DisPersion VALUES (IN TERMS OF $D L \mathrm{IN} \mathrm{ps} / \mathrm{nm}$ ) FOR 10 AND $40 \mathrm{Gbit} / \mathrm{s}$ SYSTEM FOR A GIVEN MEMORY $n_{\mathrm{mem}}$

\begin{tabular}{c|c|c}
\hline$n_{\text {mem }}$ & $10 \mathrm{Gbit} / \mathrm{s}$ & $40 \mathrm{Gbit} / \mathrm{s}$ \\
\hline \hline 1 & 928 & 58 \\
\hline 2 & 1856 & 116 \\
\hline 3 & 2785 & 174 \\
\hline 4 & 3713 & 232 \\
\hline
\end{tabular}
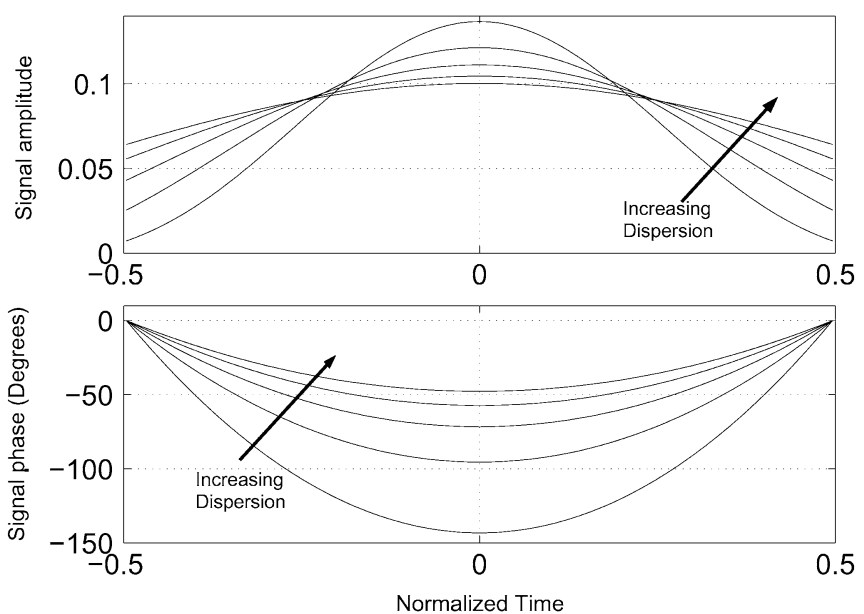

Fig. 2. Optimal pulses for $n_{\text {mem }}=1$ and $\gamma$ values ranging from 0.1 to 0.3 in 0.05 steps. Time is normalized to $T_{B}$.

$\mathrm{ETR}=0.9)$ and for the memoryless modulator $\left(n_{\mathrm{mem}}\right.$ $=1)$.

- From another point of view, if we need to transmit over a fiber with arbitrary bit rate and dispersion, we can always obtain a limited ISI condition (e.g., ETR $\geq 0.9$ ), provided that we accept a memory $n_{\text {mem }}$ at the transmitter given by

$$
n_{\text {mem }} \geq 4.241|\gamma| \Rightarrow n_{\text {mem }} \geq 8.482\left|\beta_{2} L\right| R^{2} .
$$

This is a novel and important result, stating that we can limit ISI, provided that the modulator memory $n_{\text {mem }}$ is sufficiently large, and optimal pulses are used. Table I reports the amount of accumulated dispersion that, according to (20), can be tolerated for a 10 and $40 \mathrm{Gbit} / \mathrm{s}$ system for different $n_{\text {mem }}$.

The result expressed in (20) also states that the dispersive channel, for arbitrary values of dispersion, allows an arbitrarily high bit rate, provided that $n_{\text {mem }}$ is sufficiently large and, obviously, that optimal pulses are used. Practically, as shown later in Section IV, this means that for high dispersion, the optimal pulses are compressed by the channel from an input duration $n_{\mathrm{mem}} \cdot T_{B}$ to an output duration close to $T_{\text {out }}=T_{B}$.

\section{RESULTS AND EXAMPLES OF APPLICATION}

In this section, we show some examples of application of the theory developed in the previous section. We start by showing in Fig. 2 the optimal pulses for $n_{\text {mem }}=1$ and $\gamma$ values ranging

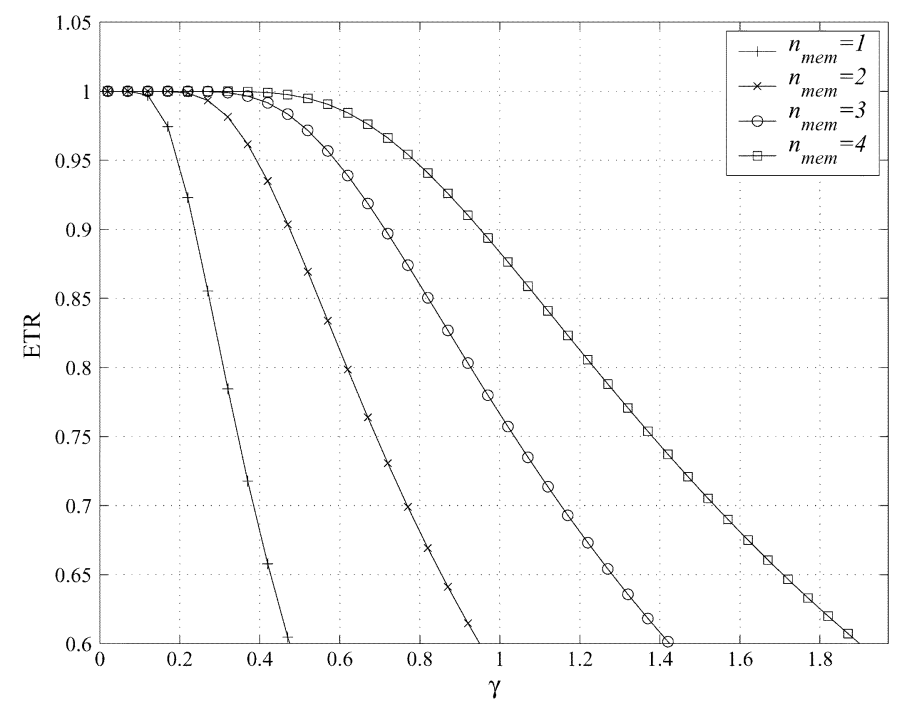

Fig. 3. ETR versus $\gamma$ for $n_{\text {mem }}=1,2,3,4$.
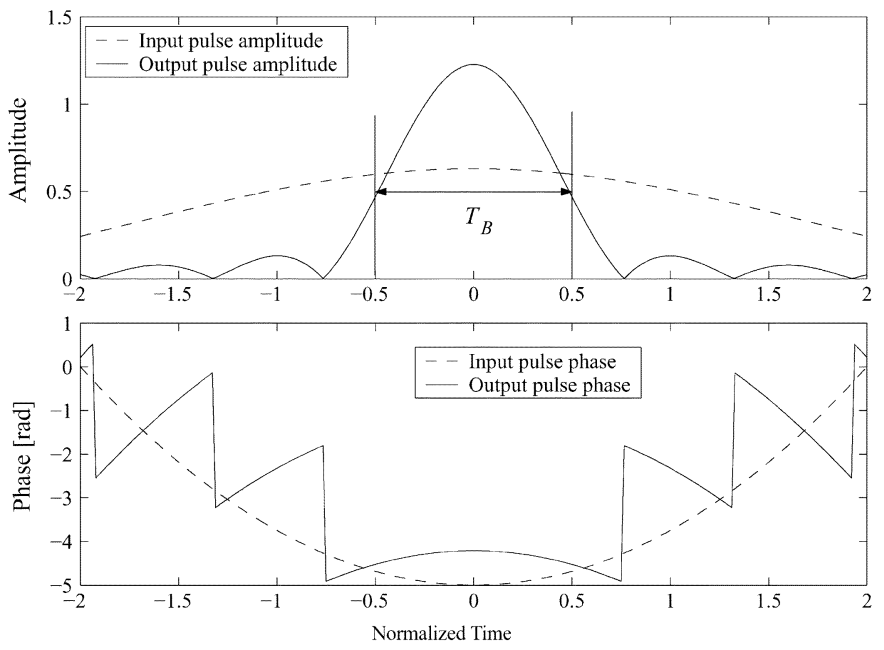

Fig. 4. Input and output signal for $n_{\text {mem }}=4$ and $\gamma=0.8$. Time is normalized to $T_{B}$.

from 0.1 to 0.3 in steps of 0.05 . The signal amplitudes show a Gaussian-like return-to-zero (RZ) shape for low values of $\gamma$, while tending to become NRZ (i.e., constant over a bit duration) for high values of $\gamma$, while the optimal signal phases show the parabolic behavior.

Fig. 3 shows the ETR versus $\gamma$ for $n_{\text {mem }}=1,2,3,4$. In particular, it shows that the amount of tolerable accumulated dispersion increases for increasing $n_{\text {mem }}$. The dispersion limit for $\mathrm{ETR}=90 \%$ is $\gamma=0.235,0.475,0.712$, and 0.95 for $n_{\mathrm{mem}}=$ $1,2,3$, and 4, respectively. A standard NRZ modulation format would have an ETR well below the optimal curve, even for $n_{\text {mem }}=1$, as we demonstrated in [6], where we showed that for NRZ, we have ETR $=0.9$ only for $\gamma=0.12$. Another interesting comparison can be done with optical duobinary, where, depending on the implementation, the maximum amount of tolerable accumulated dispersion is in the range $\gamma=[0.5,0.6]$ (see [4, Fig. 22]). Considering that the ETR limit is $\gamma=0.95$ for the optimal pulses with $n_{\text {mem }}=4$, we see that the optimal pulses can outperform optical duobinary for high values of $n_{\mathrm{mem}}$. 

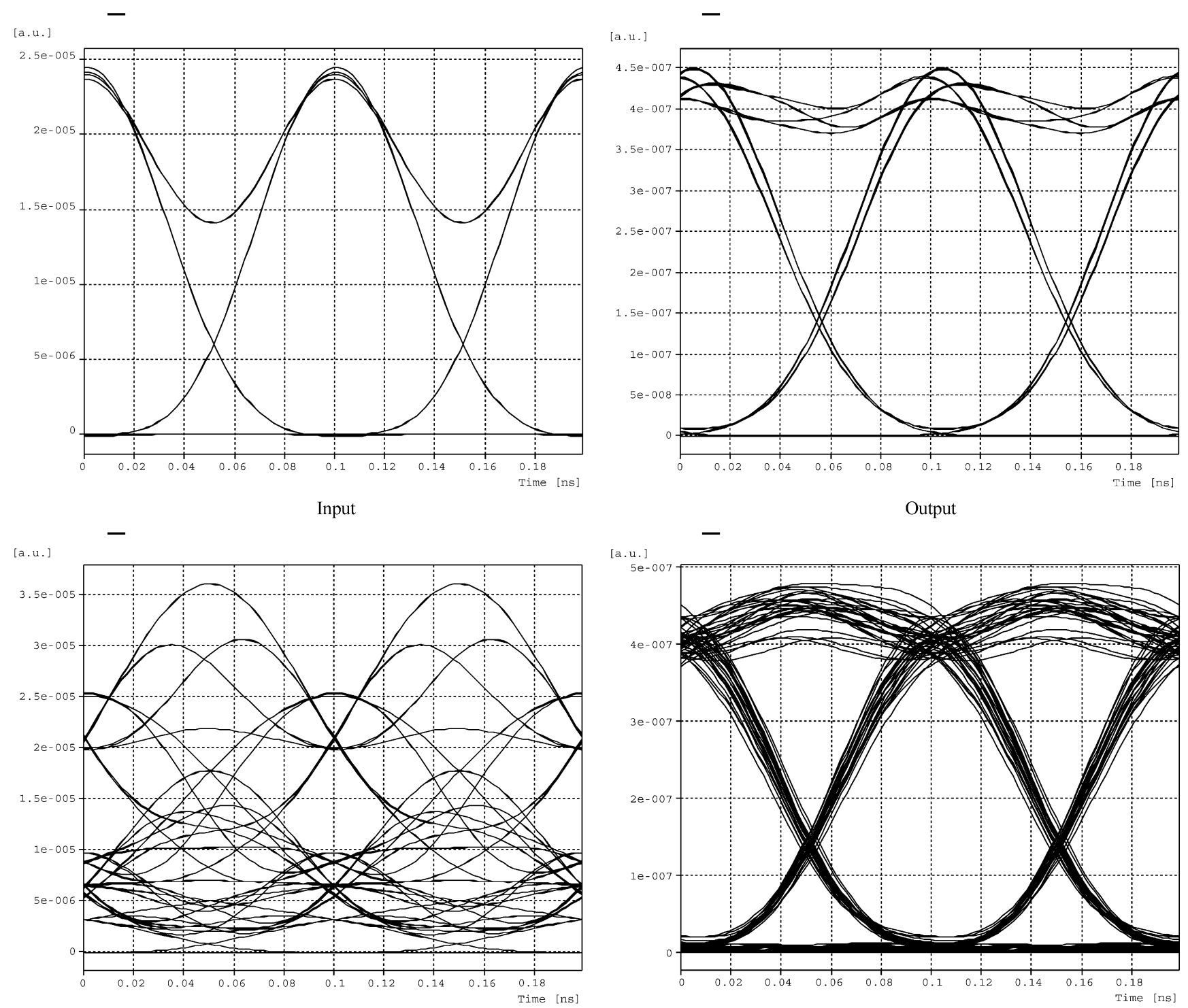

Fig. 5. Transmitted (left) and received (right) eye diagrams at $10 \mathrm{Gbit} / \mathrm{s}$ for $n_{\mathrm{mem}}=1$ (upper diagram) and $n_{\mathrm{mem}}=4$ (lower diagram) at ETR $=90 \%$ dispersion limit, corresponding to $D=928$ and $3713 \mathrm{ps} / \mathrm{nm}$, respectively (see Table I). A realistic OC-192 SONET receiver is simulated.

To better show the optimal signal behavior for $n_{\text {mem }}>1$, i.e., to understand the impact of introducing memory in the (optimal) transmitter, we show in Fig. 4 the input-output optimal signal for $n_{\text {mem }}=4$ and $\gamma=0.8$, giving an ETR $=0.94$. Note that the steps of $\pi$ in the phase are due to the sign changes in the amplitude. Thanks to the combination of the optimal chirp expressed in (13) and the optimal amplitude shape, the pulse compresses from an input duration equal to four bits, to a much lower output duration, where most of the signal energy is contained in a $T_{B}$ window.

In order to give a more practical insight on these results, and to connect the somehow theoretical performance parameter ETR to the power penalty due to dispersion, we performed a set of numerical simulations using the commercial simulator OptSim on a typical optical transmission system based on [2].

- Transmitter side: modulation at $10 \mathrm{Gbit} / \mathrm{s}$ using either optimal pulses or NRZ modulation format, with a (timedomain) raised-cosine shape, rolloff equal to 0.3 and no phase modulation (chirpless).
- Link: only dispersion has been considered, as in (1); results will be expressed as a function of the accumulated dispersion $D L$ measured in $\mathrm{ps} / \mathrm{nm}$.

- Receiver side: we considered amplified spontaneous emission (ASE) noise and a standard direct-detection receiver using a Gaussian optical filter with 3-dB bandwidth equal to $25 \mathrm{GHz}$, a photodiode, and an electrical fourth-order Bessel filter with 3-dB bandwidth equal to $7.5 \mathrm{GHz}$ (corresponding to a typical SONET-SDH OC-192 receiver [16]).

In Fig. 5, we show the corresponding eye diagrams (without noise), when using the optimal pulses for $n_{\mathrm{mem}}=1$ (upper diagram) and 4 (lower diagram) for ETR $=90 \%$ at the dispersion limits $D L=928$ and $3713 \mathrm{ps} / \mathrm{nm}$, respectively. On the left side, the transmitted eye diagrams are presented. For $n_{\text {mem }}=4$, a strong ISI is evident, as expected (see Section I-B) for a modulation with memory. On the right side, we show the received eye diagram. They are affected by low ISI in both situations, confirming that the optimization based on the ETR parameter 


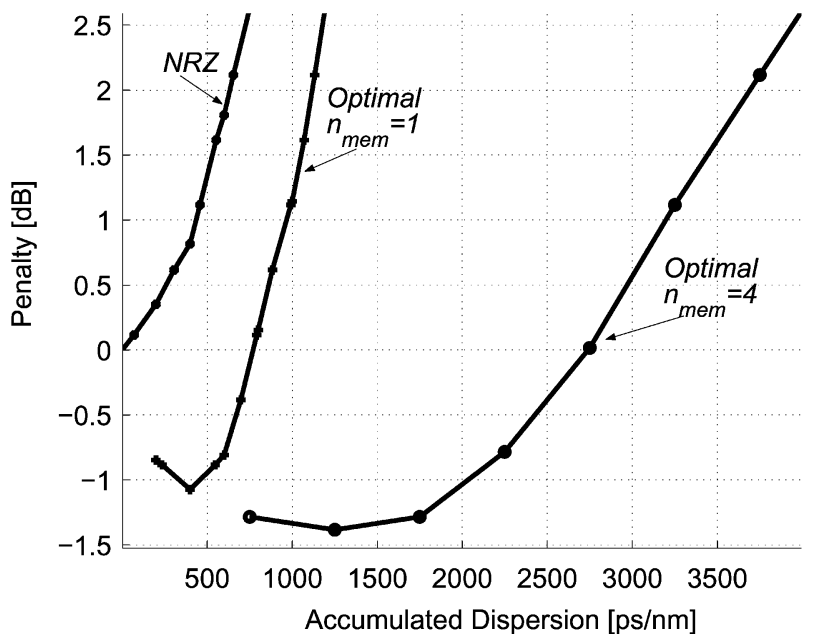

Fig. 6. Power penalty at $10 \mathrm{Gbit} / \mathrm{s}$ for NRZ, optimal pulses for $n_{\mathrm{mem}}=1$, and optimal pulses for $n_{\text {mem }}=4$. The power reference is taken as the NRZ back-to-back value.

is indeed a "good" criterion also for a realistic optical receiver and, moreover, that the ETR $=90 \%$ condition is a good choice for the definition of the dispersion limit. To further check the validity of our assumption, we perform a set of numerical simulations to estimate the actual power penalty of the systems as a function of dispersion, for the optimal signals and for standard NRZ signals. We set the reference optical SNR (OSNR) at the receiver to $15.3 \mathrm{~dB}$ over a $0.1-\mathrm{nm}$ bandwidth, giving rise, for NRZ without dispersion, to a Q-factor equal to $17.5 \mathrm{~dB}$, resulting in a bit-error probability below $10^{-12}$. This OSNR value is taken as a reference for defining the power penalty.

In Fig. 6, we plot the power penalty as a function of the accumulated dispersion for the above NRZ system, and for the optimal pulses for $n_{\text {mem }}=1$ and $n_{\text {mem }}=4$. We note the following.

- If we define, as it is commonly done, the dispersion limit at the point giving rise to a $2-\mathrm{dB}$ power penalty, we note that for the NRZ system, this limit is around $D L=650 \mathrm{ps} / \mathrm{nm}$, while for the optimized pulses for $n_{\text {mem }}=1$, it is around $1100 \mathrm{ps} / \mathrm{nm}$, and for $n_{\text {mem }}=4$, it is around $3700 \mathrm{ps} / \mathrm{nm}$. These results show the effectiveness of the optimized pulses in extending the dispersion limits. At the same time, they confirm a posteriori that the ETR optimization criterion we have chosen is valid.

- The $3700 \mathrm{ps} / \mathrm{nm}$ dispersion limit for $n_{\text {mem }}=4$ is well beyond the results that have been demonstrated for optical duobinary [8], where the limit at $10 \mathrm{Gbit} / \mathrm{s}$ is in the 2000-2500 ps/nm range, depending on the chosen system setup. Though we reckon that the optimal pulse method described in this paper does not have a simple practical implementation, we believe this result for $n_{\text {mem }}=4$ is effective in showing that the way to go to increase the dispersion limits passes through line coding with sufficiently high memory and signal chirp.

- The optimized pulses perform better than NRZ even for low dispersion values, where they show a negative penalty with respect to NRZ. This result can be explained by observing that the optimized pulse in this case (relatively low $\gamma$ ) tends to have an RZ Gaussian-like shape (as shown in Fig. 2). RZ has been shown to give an advantage over NRZ for standard optical receivers with nonmatched optical filters [2].

\section{DisCUSSION AND CONCLUSION}

We have presented a comprehensive mathematical approach to investigate the ultimate limits of the dispersion-limited optical communication channel. Our method allows deriving new bounds on the maximum bit rate achievable on the channel with limited ISI. We believe that one of our most important results is the proof that one can transmit with limited ISI over a channel with arbitrarily high dispersion, provided that the modulator memory is sufficiently high and that optimal pulses are used. The scope of this paper was mainly theoretical, even though realistic simulations have been presented in Section IV. Future work should study the potential application of these modulation formats. In particular, we are currently investigating their robustness to fiber nonlinear effects in several real-case scenarios.

\section{APPENDIX \\ DERIVATION OF INTEGRAL EQUATION (9)}

Without loss of generality, we can normalize the input pulse to have unit energy

$$
\mathcal{E}_{\text {in }}=\int_{I} s_{\text {in }}(t) s_{\text {in }}^{*}(t) d t=1 .
$$

The maximization of the ETR thus becomes a maximization of the functional $\mathcal{E}_{\text {out }}$. Since the input pulse is assumed to be strictly limited in $I$, we can write $s_{\text {out }}(t)$ as

$$
s_{\text {out }}(t)=\int_{I} s_{\text {in }}(\tau) h_{F}(t-\tau) d \tau
$$

and thus, evaluate $\mathcal{E}_{\text {out }}$ as

$$
\begin{aligned}
\mathcal{E}_{\text {out }} & =\int_{J} s_{\text {out }}(t) s_{\text {out }}^{*}(t) d t \\
& =\int_{I} \int_{I} \mathcal{K}(u, v) s_{\text {in }}(u) s_{\text {in }}^{*}(v) d u d v
\end{aligned}
$$

where the kernel is

$$
\mathcal{K}(u, v)=\int_{J} h_{F}(t-u) h_{F}^{*}(t-v) d t .
$$

Using the Hermitian scalar product notation, we can rewrite (6) and (24) as

$$
\mathcal{E}_{\text {in }}=\left\langle s_{\text {in }}, s_{\text {in }}\right\rangle \text { and } \mathcal{E}_{\text {out }}=\left\langle\mathcal{K} s_{\text {in }}, s_{\text {in }}\right\rangle .
$$

The constraint maximization problem can be solved using the Lagrange multiplier method on the functional

$$
G=\left\langle\mathcal{K} s_{\text {in }}, s_{\text {in }}\right\rangle-\lambda\left\langle s_{\text {in }}, s_{\text {in }}\right\rangle .
$$

By observing that the kernel is Hermitian, evaluating the gradient over $s_{\text {in }}$ (see, for example, [5]) and equating it to zero, we have

$$
\nabla G=\mathcal{K} s_{\text {in }}-\lambda s_{\text {in }}=0 \Rightarrow \mathcal{K} s_{\text {in }}=\lambda s_{\text {in }}
$$

which can be rewritten as (9). Finally, by multiplying both sides by $s_{\text {in }}^{*}(t)$ and integrating over $I$, we get $\mathcal{E}_{\text {out }}=\lambda \mathcal{E}_{\text {in }}$, showing 
that, for a given eigenfunction $s_{\text {in }}(t)$, the corresponding eigenvalue $\lambda$ gives the resulting ETR. Thus, the optimal solution to our problem is the eigenfunction that gives the maximum eigenvalue, i.e., the maximum ETR.

\section{REFERENCES}

[1] B. Bakhshi, M. Vaa, E. A. Golovchenko, W. W. Patterson, R. L. Maybach, and N. S. Bergano, "Comparison of CRZ, RZ and NRZ modulation formats in a $6412.3 \mathrm{~Gb} / \mathrm{s}$ WDM transmission experiment over 9000 km," in Proc. Optical Fiber Communication Conf., vol. 3, 2001, pp. WF4-1.

[2] G. Bosco, R. Gaudino, and P. Poggiolini, "An exact analysis of RZ versus NRZ sensitivity in ASE noise limited optical systems," in Proc. IEEE Eur. Conf. Optical Communication, vol. 4, Amsterdam, The Netherlands, Oct. 2001, pp. 526-527.

[3] M. Elia, G. Taricco, and E. Viterbo, "Optimal energy transfer in bandlimited communication channels," IEEE Trans. Inform. Theory, vol. 45, pp. 2020-2029, Sept. 1999

[4] E. Forestieri and G. Prati, "Novel optical line codes tolerant to fiber chromatic dispersion," IEEE J. Lightwave Technol., vol. 19, pp. 1675-1684, Nov. 2001.

[5] L. E. Franks, Signal Theory. Englewood Cliffs, NJ: Prentice-Hall, 1969.

[6] R. Gaudino and E. Viterbo, "Pulse shape optimization in dispersionlimited direct detection optical fiber links," IEEE Commun. Lett., vol. 7, pp. 552-554, Nov. 2003

[7] A. Hodzic, B. Konrad, and K. Petermann, "Alternative modulation formats in $\mathrm{N} 40 \mathrm{~Gb} / \mathrm{s}$ WDM standard fiber RZ-transmission systems," IEEE J. Lightwave Technol., vol. 20, pp. 598-607, Apr. 2002.

[8] D. Penninckx, M. Chbat, L. Pierre, and J.-P. Thiery, "The phase-shaped binary transmission (PSBT): A new technique to transmit far beyond the chromatic dispersion limit," IEEE Photon. Technol. Lett., vol. 9, pp. 259-261, Feb. 1997.

[9] J. G. Proakis, Digital Communication. New York: McGraw-Hill, 1989.

[10] M. Schilling, O. Blume, L. Nguyen, M. Schmidt, and E. Lach, "OTDM planar lightwave components (PLCs) for multiplexing from $40 \mathrm{~Gb} / \mathrm{s}$ to 80-640 Gb/s," in Proc. IEEE LEOS Annu. Meeting, vol. 2, Nov. 2002, pp. $887-888$.

[11] A. Royset, L. Bjerkam, and A. Sudbo, "Compensation of optical fiber dispersion in the electrical domain for transmission systems with direct detection," Electron. Lett., vol. 30, pp. 152-153, Jan. 1994.

[12] D. Slepian, "Some comments on Fourier analysis, uncertainty and modeling," SIAM Rev., vol. 25, no. 3, pp. 379-393, July 1982.

[13] D. Slepian and H. Pollak, "Prolate spheroidal wave functions, Fourier analysis and uncertainty-I," Bell Syst. Tech. J., vol. 40, pp. 43-63, Jan. 1961.

[14] — - "Prolate spheroidal wave functions, Fourier analysis and uncertainty-II," Bell Syst. Tech. J., vol. 40, pp. 65-84, Jan. 1961.

[15] L. E. Franks, Data Communication: Fundamentals of Baseband Transmission. New York: Halsted, 1974.
[16] Optical Interfaces for Equipment and Systems Relating to the SDH, ITU-T Recommendation G.957, July 1999.

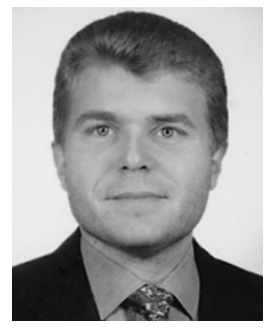

Roberto Gaudino (M'97) was born in Torino, Italy, in 1968. He received the Laurea degree and the Ph.D. degree, both in electrical engineering, from the Politecnico of Torino, Torino, Italy.

$\mathrm{He}$ is currently an Assistant Professor in the Optical Communication Group, Politecnico di Torino, where he works on several research topics related to optical communications. His main research interes is in the metro and long-haul dense wavelength division multiplexing (DWDM) systems, fiber nonlinearity, modeling of optical communication systems, and the experimental implementation of optical networks. Currently, he is in vestigating new optical modulation formats, such as polarization or phase modulation, and on packet switched optical networks. He spent the year 1997 at the Georgia Institute of Technology, Atlanta, as a Visiting Researcher in the OCPN group, where he worked in the realization of the MOSAIC optical network testbed. From 1998 to 2000, he was with the team that coordinated the development of the commercial optical system simulation software OptSim. He is author or coauthor of more than 60 papers in the field of optical fiber transmission and optical networks, is continuously involved in consulting for several companies in the optical sector, and he is also involved in professional continuing education.

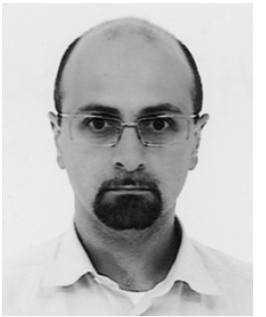

Emanuele Viterbo (M'95) was born in Torino, Italy, in 1966 . He received the Laurea degree in 1989 and the Ph.D. degree in 1995, both in electrical engineering, from the Politecnico of Torino, Torino, Italy.

From 1990 to 1992, he was with the European Patent Office, The Hague, The Netherlands, as a Patent Examiner in the field of dynamic recording, and in particular, in the field of error-control coding. Between 1995 and 1997, he held a Postdoctoral position in the Dipartimento di Elettronica, Politecnico di Torino, in communications techniques over fading channels. Between 1997 and 1998, he was a Visiting Researcher in the Information Sciences Research Center, AT\&T Research, Florham Park, NJ. Since 1998, he has been an Assistant Professor with the Politecnico di Torino, Dipartimento di Elettronica. His current research interests are in lattice codes for Gaussian and fading channels, algebraic coding theory, digital terrestrial television broadcasting, and digital magnetic recording.

Dr. Viterbo was awarded a NATO Advanced Fellowship in 1997 from the Italian National Research Council. 